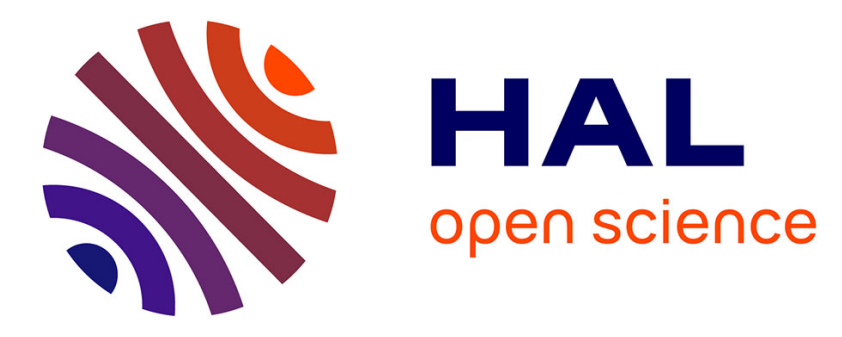

\title{
En guise de conclusion
}

Brigitte Beaujard, Michel Bonifay, Yann Codou, Marie-Geneviève Colin, Jean Guyon, Marc Heijmans, Claude Raynaud, Laurent Schneider

\section{To cite this version:}

Brigitte Beaujard, Michel Bonifay, Yann Codou, Marie-Geneviève Colin, Jean Guyon, et al.. En guise de conclusion. Gallia - Archéologie de la France antique, 2007, Antiquité tardive, haut Moyen Âge et premiers temps chrétiens en Gaule méridionale (seconde partie): monde rural, échanges et consommation, 64, pp.163-168. 10.3406/galia.2007.3309 . hal-01911690

\section{HAL Id: hal-01911690 \\ https://hal.science/hal-01911690}

Submitted on 29 Jan 2020

HAL is a multi-disciplinary open access archive for the deposit and dissemination of scientific research documents, whether they are published or not. The documents may come from teaching and research institutions in France or abroad, or from public or private research centers.
L'archive ouverte pluridisciplinaire HAL, est destinée au dépôt et à la diffusion de documents scientifiques de niveau recherche, publiés ou non, émanant des établissements d'enseignement et de recherche français ou étrangers, des laboratoires publics ou privés.

\section{(ㅇ)(1) $\$$}

Distributed under a Creative Commons Attribution - NonCommercial - NoDerivatives| 4.0 


\title{
EN GUISE DE CONCLUSION
}

\author{
Brigitte BEAUjARD, Michel BONIFAY, Yann CODOU, Marie-Geneviève COLIN, \\ Jean GUYON, Marc HEIJMANS, Claude RAYNAUd, Laurent SCHNEIDER
}

Depuis le réseau des cités jusqu’à la " culture matérielle », comme on disait naguère, nous nous sommes efforcés au fil de deux livraisons de Gallia de couvrir, ou à peu près, tous les champs frayés par les recherches récentes sur la Gaule méridionale pendant l'Antiquité tardive et le haut Moyen Âge. Une démarche aussi analytique ne se prête guère à une synthèse et moins encore à une conclusion en forme. Nulle fausse modestie, donc, dans le titre que nous avons donné à ces quelques pages par lesquelles nous souhaitons seulement achever, collectivement, de « revisiter » le midi de la Gaule à la lumière de l'exposition qui fut consacrée voici quelque vingt ans par P.-A. Février à ses « Premiers temps chrétiens » (Février, Leyge dir., 1986) car notre propos se bornera à dire en quoi, dans l'intervalle, les lignes nous paraissent avoir bougé et le paysage (scientifique, s'entend) s'être modifié.

La principale modification tient sans nul doute à l'éclairage qui est volontiers porté aujourd'hui sur l'Antiquité tardive. En fidèle disciple de H.-I. Marrou, P.-A. Février était convaincu comme son maître qu'il convenait d'appréhender la période comme « une autre Antiquité, une autre civilisation, qu'il faut apprendre à reconnaître dans son originalité, à juger par elle-même et non à travers les canons des âges antérieurs » (Marrou, 1977, p. 11). La création en 1993 de la revue Antiquité tardive n'est qu'un signe parmi d'autres que la leçon a bien été entendue - et pas seulement en France, comme en témoignent les sous-titres de cette revue, qui déclinent la formule en différentes langues: Antiguëdad Tardia, Late Antiquity, Spätantike, Tarda Antichità. Dans l'abondance des publications auxquelles cette empathie si largement partagée pour la période a donné lieu, d'autres ont pourtant vu une malheureuse « explosion " (Giardina, 1999) et l'historiographie renoue plus volontiers désormais avec la tradition ouverte voici plus de deux cents ans par E. Gibbon et son maître livre, The History of the Decline and Fall of the Roman Empire, paru en 1776.
Le titre que B. Ward-Perkins a donné à son tout récent essai, The Fall of Rome and the End of Civilization (WardPerkins, 2005), et surtout celui de la synthèse, de peu antérieure, de J. H. W. G. Liebeschuetz, The Decline and Fall of the Roman City (Liebeschuetz, 2001), offrent de ce phénomène les témoignages les plus éclairants mais sans se livrer à des clins d'œil aussi appuyés, il est bien d'autres auteurs qui participent de la même veine. Ainsi, pour ne citer que cet exemple, A. Carandini quand il a jugé dans l'introduction au volume sur L'Età tardoantica d'une Storia di Roma que : «La catastrofe del mondo antico non è una ideologia ma un fatto. Il mondo antico è finito drammaticamente alterato o addiritura sepolto, per cui vi è ben poco da rallegrarsi» (Schiavone dir., 1993, p. 12). Non que les tenants de cette thèse soient insensibles à tout ce qui apparaît comme une féconde retractatio de la tradition classique dans les transformations qui ont marqué cette «autre Antiquité " qu'était l'Antiquité tardive; pour eux cependant, "that does not mean that the concept of decline has no application to this period", comme l'a écrit J. H. W. G. Liebeschuetz en conclusion du colloque qui s'est tenu en 2003 à Munich autour de son ouvre et dont le titre, Die Stadt in der Spätantike - Niedergang oder Wandel ?, est également révélateur des inflexions récentes de la recherche (Liebeschuetz, in Krause, Witschel dir., 2006, p. 476).

Que déclin il y ait eu (et même plus) n'est pas douteux si l'on songe aux villes et autres établissements antiques ensevelis sous les sables ou retournés dans la steppe à l'état de nature comme on en connaît tant en Orient ou encore dans le «Maghreb romain ", comme aimait à dire P.-A. Février. Pour l'Occident, et singulièrement la Gaule méridionale, qui ont connu après l'effacement de Rome une tout autre histoire, il est pourtant une clé de lecture autrement opératoire ; elle tient à ce concept d'« étiage » que H.-I. Marrou avait mis en circulation dans son livre-testament, Décadence romaine ou Antiquité tardive? (Marrou, 1977, p. 147). À trente ans de distance, ce concept nous paraît largement conserver sa pertinence, comme a essayé de le montrer une commu- 
nication débattue au récent colloque de Munich (Guyon, in Krause, Witschel dir., 2006, p. 125-126) et comme le confirment implicitement les graphiques que B. Ward-Perkins a produits à l'appui d'une démonstration pourtant destinée à établir que la fin de l'Antiquité a coïncidé avec celle de la civilisation, car les creux que présentent leurs courbes à un moment ou à un autre de l'Antiquité tardive ou du haut Moyen Âge signent bien une période de basses eaux (WardPerkins, 2005, fig. 6.I, p. 124).

Que l'image d'un étiage soit venue à l'esprit d'un homme comme H.-I. Marrou, qui était avant tout un historien de la culture et de l'éducation, se comprend assez mais elle se révèle également éclairante en d'autres domaines. C'est le cas en particulier pour l'histoire institutionnelle, comme en témoigne dans la première livraison de notre dossier l'étude de B. Beaujard sur les transformations apportées à l'organigramme administratif romain qui ont conduit, pendant les derniers siècles de l'Empire et sous le règne de rois dits «barbares », à la disparition de certaines cités tandis que d'autres, au contraire, étaient promues.

L'important dans cette étude tient moins en effet à la comptabilité de ces remaniements qu'à leur caractère récurrent; il dit tout le prix que les hommes de l'Antiquité tardive ont constamment attaché au cadre institutionnel éprouvé que la cité antique leur offrait. On pourrait certes objecter que, dans le même temps, les institutions municipales avaient elles-mêmes évolué en un sens qui ne peut qu'être interprété en terme de déclin si on le mesure à l'aune du Haut-Empire, mais on ne saurait inférer de cela que le cadre dans lequel ces institutions exerçaient leur action était devenu purement formel. La preuve en est qu'il a continué à modeler le paysage du Midi (et, plus largement, de notre pays) jusqu'à la Révolution, ou presque : les limites des évêchés de l'Ancien Régime ne servent-elles pas encore aux historiens de l'Antiquité pour deviner approximativement celles des cités antiques dont ces évêchés étaient les lointains héritiers? Cette continuité, à la vérité assez relative, ne doit pas dissimuler cependant que lorsque les cités sont renées à époque romane, ce fut sous des modalités tout autres que pendant l'Antiquité; en la matière, les siècles du haut Moyen Âge (mais lesquels précisément ?) ont donc bien constitué un étiage et la question est de savoir si cette périodisation vaut également pour d'autres domaines que celui des institutions.

S'agissant du monde urbain, apporter une réponse n'est pas chose facile. Cela tient au fait qu'en dépit de la mise en garde de H.-I. Marrou, il n'est pas si aisé de « reconnaître dans son originalité, de juger par elle-même et non à travers les canons des âges antérieurs " la configuration des villes de l'Antiquité tardive. Que l'on en juge par l'exemple d'Aixen-Provence où la déprise urbaine fut tellement importante $\mathrm{au} \mathrm{III}^{\mathrm{e}}$ s. qu'on a pu conclure à un «délaissement de l'agglomération» (Nin, in Fiches dir., 1996, p. 135-154). Nul doute donc qu'Aquae Sextiae ait décliné si on se rapporte à ce qu'elle fut au Haut-Empire ; aux yeux de l'administration romaine (et certainement aussi des contemporains) la ville était pourtant restée assez importante pour qu'elle ait été promue à la fin du siècle au rang de capitale de la nouvelle province de Narbonnaise seconde née des réformes de Dioclétien. C'est assez pour changer notre regard sur elle et, plus généralement, sur les villes de Gaule méridionale pendant l'Antiquité tardive qu'il convient d'embrasser ici d'un seul coup d'œil. Cela conduit sans nul doute à faire fi de bien des nuances que l'on trouvera dans les contributions de M. Heijmans à la première livraison de ce dossier, mais c'est la rançon à payer si l'on veut esquisser une périodisation de l'évolution urbaine dans la région; au risque de simplifier beaucoup, essayons au moins de marquer quelques étapes.

«Fel. temp. Reparatio»: cette légende qui revient si fréquemment sur les monnaies $d u \mathrm{IV}^{\mathrm{e}} \mathrm{s}$. pour célébrer la restauration de l'Empire et de ses institutions, gage d'un retour espéré vers un "âge d'or », vaut sans doute aussi pour l'urbanisme au cours de ce même siècle. En témoignent, partout ou presque, l'entretien régulier et les réfections des monuments publics - y compris les temples - quand ce n'est pas, dans les villes les plus importantes comme Arles, la construction de nouveaux édifices qui ont contribué à parfaire la parure monumentale du centre civique héritée du Haut-Empire.

Les choses ont sûrement changé dès le début ou dans le courant $\mathrm{du} \mathrm{V}^{\mathrm{e}} \mathrm{s}$. selon les lieux, quand les élites municipales ont renoncé à maintenir, autant que faire se pouvait, ce décor urbain. Qu'il suffise, pour en rester à Arles, de songer à l'occupation par des particuliers de l'espace public, tant sur le forum que dans les alvéoles du cirque, de l'amphithéâtre ou du théâtre ; tout cela pour ne pas parler de l'enceinte nouvellement construite qui englobait, tel un bastion, ce dernier monument. Au même titre que les autres « enceintes tardives » de Provence ou du Sud-Ouest, ce rempart n'était pas cependant un ouvrage construit à la hâte et de médiocre facture : c'est peut-être à lui qu'était destinée, au début du siècle suivant, la donation faite par Théodoric aux Arlésiens «pour qu'ils restaurent leurs murailles dans leur antique splendeur ». 
La formule fait écho à la légende monétaire que l'on a évoquée à propos du $\mathrm{IV}^{\mathrm{e}} \mathrm{s}$. mais quand nos connaissances sur les villes $d u \mathrm{VI}^{\mathrm{e}} \mathrm{s}$. et des siècles ultérieurs sont des plus courtes, le danger serait de lui accorder un trop grand crédit. Car que les nouveaux maîtres aient eu pour souci cette temporum reparatio qui avait été avant eux celui de l'Empire finissant est une chose ; que leurs efforts aient été couronnés de succès en est une autre. Nul doute en effet qu'en cette aube du haut Moyen Âge, le tissu urbain continuait à se défaire, au même titre que la parure monumentale héritée de Rome (ou ce qu'il en restait), mais nous sommes bien en peine de marquer au sein de cette évolution une césure aussi affirmée que celle $d u \mathrm{~V}^{\mathrm{e}} \mathrm{s}$. Cela est si vrai que, comme en matière d'institutions, il est difficile de préciser quand l'étiage a été atteint pour l'histoire urbaine du Midi : sans doute au cours de ces Dark Ages que constituent encore pour nous les $\mathrm{IX}^{\mathrm{e}}$ et $\mathrm{X}^{\mathrm{e}} \mathrm{s}$. pendant lesquels les villes paraissent avoir été réduites à très peu de choses, comme l'ont montré par exemple les fouilles autour du groupe épiscopal de Fréjus (Février et al., 1985).

Le temps de la campagne était-il le même que celui des villes? La question doit être posée autrement que dans les années 1980 où l'on ne savait à peu près rien de l'habitat rural de l'Antiquité tardive et du haut Moyen Âge dans le sud de la France, de sorte que le postulat d'un déclin démographique, d'un recul des terres cultivées reposait plus sur l'absence de données archéologiques et la rareté des sources écrites que sur des arguments solides. Vingt années de recherches soutenues ont depuis apporté des matériaux de plus en plus nombreux issus de fouilles ou de «territoiresateliers » finement prospectés, qui interdisent de considérer le monde des campagnes comme un ensemble monolithique relevant d'une trajectoire unique.

Ce nouveau regard participe d'une historiographie qui remonte au moins à $\mathrm{N}$. Baynes, pour qui toute interprétation solide du «déclin " postulé devait passer par l'identification préalable des differentiae entre les régions plus ou moins riches (Baynes, 1943). Dans le même sens, R. Mac Mullen a avancé plus récemment qu'aucune explication du déclin, et du terme lui-même, « ne peut prétendre à la moindre validité si elle ne vise pas et si elle ne concourt pas à éclairer la situation de l'Empire dans son ensemble » (Mac Mullen, 2004, p. 95). L'agriculture a décliné ou pris son essor dans tel secteur mais pas dans tel autre, sans qu'il soit nécessaire de s'interroger sur une éventuelle ruine définitive. Le récent ouvrage de C. Wickham apporte de ce phénomène la plus belle des démonstrations, non parce qu'il est bâti sur le remplacement d'interprétations obsolètes par de nouvelles généralisations hâtives, mais parce qu'il évite le recours à des antithèses simplistes comme celles de la catastrophe et de la continuité. À l'échelle euro-méditerranéenne, il permet désormais d'entrevoir le monde de l'Antiquité tardive comme un monde de régions, sinon de microrégions (Wickham, 2005).

Chaque région avec ses contextes spécifiques eut de fait sa propre histoire et, à l'intérieur de celle-ci, les modes et les dynamiques de peuplement pouvaient également différer d'un canton à l'autre. Ainsi, dans la cité d'Arles des Ve-VII ${ }^{\mathrm{e}} \mathrm{s}$., le secteur de l'étang de Berre prit un nouvel essor autour de la place forte d'Ugium tandis que sur la rive droite du Rhône, autour du castrum d'Ugernum/Beaucaire, le système des villae paraît quant à lui s'effondrer dès le $\mathrm{V}^{\mathrm{e}} \mathrm{s}$. Aussi le temps de la campagne ne peut-il se mesurer avec les mêmes instruments que celui des villes où « la sauvegarde par l'Église de ses archives et de son patrimoine bâti permet, dans le seul domaine ecclésiastique, de suivre la genèse d'une topographie complexe qui à son tour continue de légitimer le poids de la tradition, les lieux d'exercice du pouvoir et les organes de contrôle de la société, quels qu'aient été les changements politiques »(Galinié, 2006, p. 104). Le quasi-monopole des cités dans les sources écrites, dans l'architecture monumentale civile ou religieuse (mais aussi, jusqu'à une date récente, dans les interventions archéologiques), fait pourtant que l'on a trop longtemps continué de regarder les campagnes $\mathrm{du}$ haut de la pyramide institutionnelle selon la grille de lecture que proposent les textes (Fixot, 2000).

Inverser le regard exige sans doute un labeur infini tant la diversité de destins de chaque canton rural peut être grande, tant la collecte incommode des données nécessite modestie et persévérance. C'est néanmoins dans cette voie que se sont engagés les travaux d'archéologie rurale dans le Midi depuis ces deux dernières décennies, travaux qui marquent en définitive l'émergence d'une archéologie non classique. Ce n'est pas là céder à une pointilleuse minutie qui ferait perdre de vue toute possibilité de généralisation ; cela relève d'une démarche qui tente de prendre la mesure et la complexité des variations régionales à partir de la production de sources archéologiques sans chercher à parler de l'essor ou du déclin dans l'Empire tout court.

Il ressort de ces enquêtes précises que le destin des unités du peuplement rural du Midi méditerranéen s'est inscrit plusieurs siècles durant dans les contrecoups de la croissance brutale du I ${ }^{\mathrm{er}} \mathrm{s}$. C'est en quelque sorte « l'onde de choc » de cette croissance exceptionnelle qui est susceptible d'expliquer en partie les cycles d'abandons des établisse- 
ments ruraux dans la seconde moitié du $\mathrm{II}^{\mathrm{e}} \mathrm{s}$. et au $\mathrm{III}^{\mathrm{e}} \mathrm{s}$. Dans cette perspective, les convulsions du système que l'on croit pouvoir détecter grâce au tarissement des créations d'exploitation ne sauraient être interprétées en termes de «déclin» ou de «crise » généralisée, mais comme le remaillage des réseaux d'exploitations et d'habitats. Cette sélection par touches successives a conduit à la survie d'établissements solides au détriment d'installations fragiles; la nouvelle hiérarchie à laquelle elle a donné naissance témoignerait finalement du seuil de maturité que le système agraire de la Gaule méridionale avait atteint au IV $\mathrm{e}$.

Mais ce que la recherche récente fait peut-être mieux percevoir aujourd'hui tient surtout à l'identification d'une nouvelle phase de développement inscrite entre la seconde moitié $d u \mathrm{~V}^{\mathrm{e}} \mathrm{s}$. et le premier tiers $\mathrm{du} \mathrm{VI}^{\mathrm{e}} \mathrm{s}$. L'augmentation des créations d'établissements paraît traduire une intensification de la vie rurale et signe surtout la mise en place d'une nouvelle géographie des campagnes, qui s'étoffe alors et s'affranchit des cadres et des trajectoires légués par le HautEmpire. De nouveaux espaces sont investis et la vie rurale s'enracine pour longtemps autour de centres ruraux et de formations sociales appelés à traverser tout le haut Moyen Âge dans le cadre territorial de ce que les sources finissent par désigner comme une villa. Malgré des cycles de développement inégaux d'une microrégion à l'autre, l'empreinte du $\mathrm{VI}^{\mathrm{e}} \mathrm{s}$. inscrit désormais l'habitat rural au cour d'un espace géographique qui lui est propre et les familles paysannes dans des groupes sociaux de structure locale.

Cette intensification de la vie rurale que l'on croit pouvoir détecter entre les années 450 et 550 se mesure aussi et surtout dans la Gaule du Sud-Est par l'émergence de nouvelles places rurales perchées et fortifiées, beaucoup plus nombreuses que ce qui avait été entrevu il y a une vingtaine d'années. Ces «oppida tardifs » ou, si l’on préfère, ces castra et castella sont le signe possible d'une autre forme d'encadrement de l'espace rural, sinon de l'émergence d'un nouveau «niveau de ville». Presque toujours ignorées des sources écrites, ces places anonymes marquent la révélation archéologique d'un phénomène d'une importance cardinale, même si nous n'en avons pour l'instant qu'une appréhension partielle. Sa mise en évidence ouvre dès à présent la voie à des considérations plus approfondies sur le thème de l'insécurité, des capacités défensives du monde rural et peut-être plus largement encore sur un nouveau «mitage " de l'organisation territoriale qui a mis fin au régime de la cité antique en perturbant les anciennes hiérarchies, en introduisant cette distanciation proprement médiévale qui fait que noyau urbain et milieu rural cessent d'être indissociables. Il reste beaucoup à faire, mais des jalons existent maintenant pour opposer à l'apparente somnolence de certains chefs-lieux de cités du Midi pendant le haut Moyen Âge les destins divers de ces castella et castra ruraux jusqu'à l'époque carolingienne. C'est dire qu'en la matière, la période a moins été marquée par un étiage que par une profonde « redistribution des cartes».

Les échanges, dont les objets de céramique et de verre nous renvoient probablement une image assez biaisée, ont donné lieu à une autre « redistribution des cartes » pour laquelle point n'est besoin d'avoir recours à la notion de « restauration des temps passés ». Les arrivages de denrées méditerranéennes en Gaule du Sud semblent ne subir aucun fléchissement au IV ${ }^{\mathrm{e}} \mathrm{s}$. Bien au contraire, c'est à ce moment-là que les amphores et la vaisselle sigillée africaine connaissent leur plus grande diffusion tandis que le siècle suivant consacre un véritable «boom » dans les importations de vins de Méditerranée orientale, dont les textes se font encore l'écho à l'époque de Grégoire de Tours. Cette persistance du commerce méditerranéen en Gaule du Sud durant l'Antiquité tardive avait été mise en évidence dès 1986 mais les recherches récentes ont révélé son exceptionnelle durée. Il est désormais possible de suivre les arrivages d'amphores africaines et orientales jusqu'à la fin du VII ${ }^{\mathrm{e}}$ s. ou au début du VIII ${ }^{\mathrm{e}}$ s. non seulement dans des grandes villes, comme Marseille, Lyon et Bordeaux, mais également en milieu rural, comme au mont Bouquet ou à Dassargues. Ainsi, pas plus l'installation des royaumes barbares en Gaule du Sud que la conquête arabe au Proche-Orient et en Afrique ne semblent, à elles seules, avoir eu raison des grands courants commerciaux hérités du monde romain.

Cependant, on ne peut rejeter en ce cas encore toute idée de déclin ou, tout au moins, refuser d'admettre que les mécanismes commerciaux ont subi durant l'Antiquité tardive une profonde métamorphose. S'il est évidemment impossible de jauger le volume réel de ces échanges, il semble toutefois qu'aux $\mathrm{V}^{\mathrm{e}}$ et $\mathrm{VI}^{\mathrm{e}} \mathrm{s}$., les denrées africaines et orientales irriguent plus faiblement que par le passé l'intérieur des terres. Certes, un bon nombre d'habitats de hauteur créés dans la seconde moitié du V $\mathrm{V}^{\mathrm{e}} \mathrm{s}$., même éloignés des côtes et encore de nos jours difficiles d'accès, semblent maintenir un niveau de vie proche de celui des grandes villae de plaine de l'époque romaine tardive, avec une proportion non négligeable d'arrivages africains et orientaux (amphores et vaisselle). Ce sont d'ailleurs ces objets, dont la typologie est constante des Pyrénées aux 
Alpes, qui permettent le mieux de saisir la contemporanéité du phénomène de «perchement » de l'habitat et son acmé dans le deuxième quart $d u \mathrm{VI}^{\mathrm{e}} \mathrm{s}$. Mais ces grands plats de sigillées africaines utilisés dans de modestes intérieurs au sol de terre battue et ces amphores qui avaient été distribuées jusque sur les barres calcaires du Midi languedocien et provençal ne doivent pas faire oublier qu'en d'autres régions, en Aquitaine notamment, la vaisselle d'importation et le vin oriental sont alors devenus, en raison de leur rareté, l'apanage des élites civiles et religieuses. L'archéologie urbaine, jouant quant à elle sur de plus grandes masses de mobiliers, révèle à partir du milieu $d u \mathrm{~V}^{\mathrm{e}} \mathrm{s}$. des tendances plus complexes : disparition des produits hispaniques, augmentation des importations orientales d'amphores et de vaisselle, fléchissement des arrivages africains.

La chute des importations de sigillées africaines, générale en Méditerranée durant la seconde moitié du V ${ }^{\mathrm{e}} \mathrm{s}$, en même temps qu'elle paraît avoir favorisé le renouveau éphémère des productions de céramiques fines provençales, est peut-être tout simplement une manifestation de la fin du système annonaire. Il paraît de plus en plus probable que la diffusion capillaire des vaisselles de Tunisie septentrionale et centrale était en fait liée à l'approvisionnement en blé de Rome et de l'Empire. En effet, tout comme le blé, la sigillée africaine est invisible dans l'archéologie sous-marine des navires africains des $\mathrm{II}^{\mathrm{e}}-\mathrm{IV}^{\mathrm{e}} \mathrm{s}$. En revanche, en même temps qu'elle se raréfie (relativement) dans les habitats de la Gaule du Sud, la vaisselle africaine apparaît dans le chargement des navires marchands du milieu du $\mathrm{V}^{\mathrm{e}} \mathrm{s}$., désormais signalés aux archéologues par une cargaison principale d'amphores (épave Dramont E).

Dans le domaine des échanges comme dans la plupart des autres domaines, la césure principale semble donc se produire au cours du $\mathrm{V}^{\mathrm{e}} \mathrm{s}$. Le grand commerce subsiste en Gaule du Sud aux VI ${ }^{\mathrm{e}}$ et VII ${ }^{\mathrm{e}}$ s., mais il obéit sans doute à de nouvelles règles. Au début du VIII ${ }^{\mathrm{e}}$ s., pourtant, le niveau des échanges a probablement atteint ici son étiage. C'en est fini de cette mondialisation à l'antique qui supposait qu'un Sidoine Apollinaire s'excusât de ne pas offrir à son hôte un vin de Gaza et que la production des verriers de Maguelone et de Marseille fût dépendante de matières premières importées de Palestine ou d'Égypte. L'arrêt des importations d'objets manufacturés entraîne également une rupture dans les échelles chronologiques du midi de la France aux VIII ${ }^{\mathrm{e}}$ et $\mathrm{IX}^{\mathrm{e}} \mathrm{s}$., période dont on doit admettre qu'elle mérite encore, du strict point de vue des typologies de céramiques et de verres, son appellation de Dark Ages.
S'agissant d'un dossier dont les derniers mots du titre sont «les premiers temps chrétiens", on dira pour finir quelques mots du processus de christianisation, d'autant que, par souci peut-être de marquer ses distances avec cette «new and rosy Late Antiquity » contre laquelle B. WardPerkins et quelques autres ont brillamment rompu des lances, la recherche récente tend sinon à en minimiser l'importance, du moins à le cantonner au seul domaine de l'« histoire des mentalités ", comme on disait naguère (Ward-Perkins, 2005, p. 169-183). Mais même pour ceux qui prennent pleinement en compte le phénomène, les lignes ont quelque peu bougé, le paysage de la recherche s'est légèrement modifié depuis vingt ans en ce domaine aussi.

Cela tient essentiellement au fait que les fouilles et recherches récentes conduisent de façon sans cesse plus convaincante à envisager le processus sous le signe de la très longue durée. Le cas est patent pour les pratiques qui entouraient la mort car même si un lien fort tend à s'établir à partir $\mathrm{du} \mathrm{V}^{\mathrm{e}} \mathrm{s}$. entre église et sépulture, la démonstration paraît faite désormais que la naissance d'un «cimetière chrétien » est à placer au plus tôt à l'époque carolingienne (Treffort, 1996b ; Rebillard, 2003 ; Lauwers, 2005a), l'Antiquité tardive et le haut Moyen Âge n'ayant jamais connu à proprement parler une «mort chrétienne », mais seulement des « morts de chrétiens ». Encore les signes qui marquaient les tombes de ces fidèles défunts sont-ils souvent restés assez discrets au sein des nécropoles des $\mathrm{III}^{\mathrm{e}}-\mathrm{VII}{ }^{\mathrm{e}}$ s., comme le montre dans la première livraison de ce dossier la contribution de C. Raynaud. Mais il n'est pas jusqu'au phénomène de l'émergence dans le Midi d'un paysage christianisé qui ne doive être plus soigneusement pesé désormais à la lumière de l'enquête sur la Topographie chrétienne des cités de la Gaule (TCCG) ou des approches synthétiques récentes sur les paroisses rurales (Delaplace dir., 2005),

Même dans les secteurs, pourtant assez nombreux en Gaule méridionale, qui ont été précocement touchés par la mission chrétienne, les premiers signes monumentaux de la nouvelle foi sont assez tardifs en effet, puisque rarissimes sont ceux que l'on peut dater à coup sûr avant 400. Il a fallu du temps pour que les fidèles et leurs pasteurs aient à la fois le désir, les moyens financiers et le poids politique qui leur permît de s'afficher ainsi aux yeux de leurs contemporains, ce qui ne saurait surprendre si l'on songe que jusqu'à la fin $\mathrm{du} \mathrm{IV}^{\mathrm{e}} \mathrm{s}$. le christianisme entrait encore en concurrence avec la religion officielle de l'Empire. Et même si une fois engagé, ce processus de conquête de l'espace par la nouvelle foi n’a plus cessé, il a procédé par vagues successives qui se 
sont prolongées, dans les campagnes comme dans les villes, au-delà du terme chronologique retenu pour ce dossier.

Tout cela sans se départir du pragmatisme qui est une des constantes de l'histoire ecclésiastique car si dans le monde urbain les édifices liés à la nouvelle foi n'ont constitué (du moins au début) qu'un ultime apport à la parure monumentale des chefs-lieux, dans les campagnes, la constitution d'un réseau d'établissements chrétiens a participé au contraire de cette «nouvelle donne» qui fut un trait distinctif de la période. La construction, sur le modèle des groupes épiscopaux des chefs-lieux, de ce que l'on est enclin à interpréter comme des «groupes presbytéraux » en est un signe car ces monuments constituaient pour les fidèles autant de possibles éléments d'identification, d'appartenance à une communauté. Au sein du monde rural, l'érection d'églises paroissiales (mais aussi d'autres établissements, funéraires ou cultuels) esquissait ainsi une géographie qui ne coïncidait pas nécessairement avec celle qui était en usage sous l'Empire. Ce n'était pourtant là qu'une esquisse car le statut proprement territorial des paroisses rurales, tel que l'entendra le Moyen Âge, était encore largement inchoatif pendant l'Antiquité tardive.

Qu'en matière de topographie chrétienne, le $\mathrm{V}^{\mathrm{e}} \mathrm{s}$. ait ainsi marqué un tournant ne manque pas d'interroger si l'on se souvient que ce siècle a également coïncidé avec la seule césure que l'on puisse marquer avec certitude dans les différents domaines que nous avons abordés dans ce dossier. Faut-il voir là un hasard ? Non, sans doute, si l'on s'avise que les pratiques de l'évergésie ont connu une véritable conver- sion - dans tous les sens de ce terme - dès les débuts l'Antiquité tardive, comme l'ont montré les rapports conjugués de C. Lepelley, C. Roueché, Y. Duval et L. Pietri au congrès international d'épigraphie de Nîmes de 1992 (Christol, Masson dir., 1997, p. 335-396). Donnant à leur générosité un sens tout autre que leurs ancêtres, les notables acquis à la nouvelle foi ont dès lors privilégié les fondations pieuses au détriment de l'entretien, la rénovation ou la construction des autres monuments publics de leur cité. Et cela n'a pu que favoriser l'appropriation de ces édifices par des particuliers qui ont installé sur leur emprise un habitat «parasitaire » ou encore la spoliation de leur décor, au profit notamment des édifices de culte chrétiens.

Dès la première moitié du $\mathrm{V}^{\mathrm{e}} \mathrm{s}$., Honorat de Marseille campe ainsi un clerc arlésien occupé à dépouiller le théâtre antique de la ville sur l'ordre de l'évêque Hilaire (Vie de saint Hilaire, 20). D'aucuns verraient là une illustration du mot fameux par lequel A. Piganiol avait choisi de clore son Empire chrétien: "La civilisation romaine n'est pas morte de sa belle mort. Elle a été assassinée » (Piganiol, 1972, p. 466, significativement repris par Ward-Perkins, 2005, p. 173). Mais - que B. Ward-Perkins nous le pardonne - ce n'est ni par docilité envers la Fondation européenne de la science et son thème de recherche «The Transformation of the Roman World», ni parce que nous en partageons l'idéologie implicite qu'il a dénoncée (ibid., p. 174) que nous nous montrerons plus nuancés: disons que s'amorçait là - mais s'amorçait seulement - le lent glissement de la cité antique à la chrétienté médiévale qui fut le fait de l'Antiquité tardive et du haut Moyen Âge. 